\title{
Autoeficacia y percepción de control en la prevención de la enfermedad cardiovascular"
}

\section{Self-Efficacy and Perceived Control in the Prevention of Cardiovascular Disease}

Recibido: 30 de junio de 2009 | Revisado: 6 de octubre de 2009 Aceptado: 20 de noviembre de 2009

\author{
AMPARO CARPI BALlester ${ }^{* *}$ \\ Universitat Jaume I, España \\ Pilar González NAVARro*** \\ ROSARIO ZURRIAGA LLORENS \\ Universidad de Valencia, España \\ JuAN CARLOS MARZO CAMPOS ${ }^{* * * *}$ \\ Universidad Miguel Hernández de Elche, España \\ ABRAHAM P. BUUNK ${ }^{* * * * *}$ \\ Universidad de Groningen, Holanda \\ Royal Netherlands Academy of Science, Holanda
}

Para citar este artículo. Carpi Ballester, A., González Navarro, P., Zurriaga Llorens, R., Marzo Campos, J.C., \& Buunk, A.P. (2010). Autoeficacia y percepción de control en la prevención de la enfermedad cardiovascular. Universitas Psychologica, 9 (2), 423. 432.

* Este estudio ha sido desarrollado en el marco del proyecto CONSOLIDER (SEJ2006-14086) financiado por el Ministerio de Educación y Ciencia de España.

* Departamento de Psicología Básica, Clínica y Psicobiología. Facultad de Ciencias Humanas y Sociales. Avda. Sos Baynat, s/n. 12071 Castellón (España). Correo electrónico: carpib@psb.uji.es

*** Departamento de Psicología Social. Facultad de Psicología. Avd. Blasco Ibañez, 21. 46010 Valencia (España).Correos electrónicos: Rosario.Zurriaga@ uv.es,Pilar.Glez-Navarro@uv.es\

${ }^{* * * * *}$ Departamento de Psicología de la Salud. Avd. Universidad S/N. Edificio Altamira. 03202 Elche (España).Correo electrónico: jc.marzo@umh.es

******* Psychology, University of Groningen. Griote Kruisstraat 2/1.9712 TS Groningen (Netherlands). Correo electrónico: a.p.buunk@rug.nl.
RESUMEN

Desde la Teoría de Acción Planeada (TAP), el objetivo de este trabajo es analizar si la autoeficacia y la percepción de control presentan diferencias predictivas sobre la intención y las conductas preventivas de la enfermedad cardiovascular. La muestra estuvo compuesta por 359 sujetos. Los datos han sido analizados empleando el paquete estadístico EQS 6.1. Los resultados muestran que la autoeficacia influye positiva y significativamente sobre la intención y sobre la conducta, mientras que la percepción de control influye negativa y significativamente sobre la intención de conducta, pero no sobre la conducta preventiva. Este trabajo ha mostrado la conveniencia de distinguir entre autoeficacia y percepción de control en la TAP en la prevención de conductas de salud cardiovascular.

Palabras clave autores

Autoeficacia, percepción de control, prevención, enfermedad cardiovascular, estudio descriptivo mediante encuesta.

Palabras clave descriptores

Autoeficacia, enfermedades cardiovasculares, adaptación (psicología).

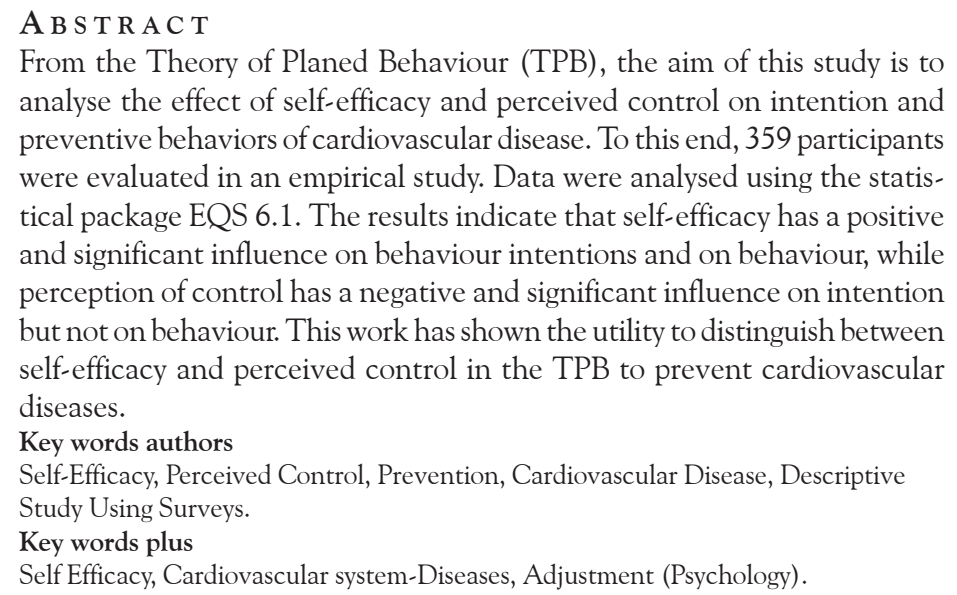

From the Theory of Planed Behaviour (TPB), the aim of this study is to analyse the effect of self-efficacy and perceived control on intention and preventive behaviors of cardiovascular disease. To this end, 359 participants were evaluated in an empirical study. Data were analysed using the statistical package EQS 6.1. The results indicate that self-efficacy has a positive and significant influence on behaviour intentions and on behaviour, while perception of control has a negative and significant influence on intention but not on behaviour. This work has shown the utility to distinguish between self-efficacy and perceived control in the TPB to prevent cardiovascular diseases.

Key words authors

Self-Efficacy, Perceived Control, Prevention, Cardiovascular Disease, Descriptive Study Using Surveys.

Key words plus

Self Efficacy, Cardiovascular system-Diseases, Adjustment (Psychology). 


\section{Introducción}

Entre las principales causas de morbilidad y mortalidad en las sociedades industrializadas se encuentran el conjunto de las enfermedades cardiovasculares. Estas enfermedades del sistema circulatorio se clasifican en cuatro tipos: enfermedades isquémicas del corazón, enfermedades cerebrovasculares, enfermedades vasculares periféricas y otras enfermedades cardiovasculares (Corella \& Ordovás, 2007).

Las causas y los factores de riesgo que desencadenan este tipo de trastornos son diversos. Entre los más tradicionales y generales se citan: los antecedentes familiares hereditarios, los antecedentes personales, los antecedentes conductuales y los factores socioculturales (Navas, Sánchez \& Molero, 1990). Como señalan diversos autores (Armitage, 2005; Burnout \& Escobar, 2000; Fernández-Abascal, Martín \& Domínguez, 2003; Jhonston, Jhonston, Pollard, Kinmonth \& Mant, 2004), los antecedentes conductuales como son: comer en exceso, tomar alimentos ricos en grasas animales, consumir tabaco y alcohol, la escasa realización de ejercicio físico, el afrontamiento inadecuado de las situaciones de estrés y su consiguiente alteración emocional, pueden contribuir al desarrollo de la enfermedad cardiovascular. Esto se debe a que contribuyen a la formación de la placa de ateroma y la consiguiente obstrucción de los vasos sanguíneos, dificultando y aumentando el esfuerzo cardiaco en el bombeo de la sangre y su consiguiente transporte de oxigeno y alimento a los distintos órganos y sistemas corporales.

De hecho, como señala la OMS en su informe "Global Health Risk" (2009) entre los factores de riesgo que explican el 75\% de las enfermedades cardiovasculares que aparecen cada año, se encuentran entre otros: el consumo de tabaco, la ausencia de ejercicio físico y el tipo de alimentación. Estos factores están relacionados con estilos de vida poco saludables y, aunque se puede intervenir sobre ellos ya que son modificables, son responsables de una reducción de la esperanza de vida en los países más avanzados.

Los sistemas sanitarios dedican grandes esfuerzos para facilitar la adopción de conductas que eviten el riesgo controlable de la enfermedad cardiovascular. No obstante, la evidencia sugiere que estos esfuerzos son necesarios pero no suficientes para promover cambios en las conductas de las personas (Myers \& Frost, 2002) sobre todo si los beneficios sobre la salud no son inminentes (Conner, 2008).

Un modelo diseñado y ampliamente investigado para conocer y predecir las variables que sustentan los comportamientos relacionados con la salud es la Teoría de la Acción Planeada -TAP (Ajzen, 1985, 1991), una extensión de la Teoría de la Acción Razonada - TAR (Ajzen \& Fisbein, 1980). Estos dos modelos comparten las mismas variables (la actitud, la norma subjetiva y la intención de conducta) con excepción de la percepción de control que fue incorporada por Ajzen (1985), para mejorar el valor predictivo del modelo predecesor. Entre las conductas de prevención de la salud estudiadas desde este marco teórico están las relacionadas con el ejercicio y la actividad física (Armitage, 2005; Brenes, Strube \& Storandt, 1998; Courneya, Conner \& Rhodes, 2006), el uso del preservativo (Albarracín, Johnson, Fishbein \& Muellerleile, 2001; Heeren, Jemmott III, Mandeya \& Tyler, 2007), el consumo de tabaco (Conner, Sandberg, McMillan \& Higgins, 2006; Panday, Reddy, Ruiter, Bergström \& De Vries, 2007), la ingesta de grasas (Armitage \& Conner, 2002), adhesión a tratamientos (Holguín, Correa, Arrivillaga, Cáceres \& Varela, 2006) y la enfermedad cardiovascular (Carpi, Zurriaga, González, Marzo \& Buunk, 2007; Johnston et al., 2004).

Los determinantes directos de cualquier acción siguiendo la TAP son la intención y la percepción de control. La intención es la decisión o el propósito firme de realizar una conducta en un futuro próximo inmediato y por ello un precursor inmediato de la acción. La percepción de control es una evaluación sobre la ejecución de una conducta atendiendo tanto a factores externos (facilidad o dificultad para realizar una acción) como internos (capacidad y/o habilidad para realizar la conducta). La percepción de control tiene implicaciones directas sobre la intención, ya que cuando una persona cree tener las oportunidades o recursos 
para realizar cierta conducta, es más probable que tenga también la intención de realizarla. Por el contrario, si la personas no cree tener esas oportunidades o recursos, es bastante improbable que surja la intención de realizar la conducta.

Por lo tanto, la ejecución de una conducta no depende sólo de la intención para realizarla sino también del control que la persona piensa que tiene sobre su ejecución. De esta forma, la percepción de control puede influir indirectamente sobre la conducta a través de la intención, y también directamente sobre la conducta cuando la persona cree tener control real sobre la misma (Ajzen, 1988).

La percepción de control ha sido relacionada con la autoeficacia debido a las similitudes conceptuales y predictivas que presentan. Para Ajzen y Timko (1986), la autoeficacia y la percepción de control, tal y como son definidas en la TAP, podrían ser utilizadas indistintamente por las características comunes de los factores que las definen.

Sin embargo, mientras que la percepción de control de la TAP incluye tanto factores internos (percibirse con habilidades, capacidades para la acción) como externos (obstáculos en la ejecución a causa de otras personas, falta de oportunidad en cuanto a tiempo y lugar para ejecutar la conducta, etc.), la autoeficacia (Bandura, 1977) hace referencia principalmente a variables internas al sujeto, es decir, a la percepción de habilidad o capacidad para realizar los cambios requeridos en los comportamientos propuestos.

En este sentido, Terry (1993) y Terry y O'Leary (1995) sugieren la necesidad de etiquetar de forma diferente el control interno (capacidad) como autoeficacia y el control externo (facilidad-dificultad percibida) como percepción de control, recogiendo la definición que realizaron Ajzen y Madden (1985). Los resultados de sus trabajos informaron que la autoeficacia predijo mejor y de modo significativo e independiente la intención, mientras que el valor predictivo de la percepción de control (percepción de obstáculos) fue más débil.

Con objeto de analizar si la percepción de control y la autoeficacia presentan vías de influencia directas e indirectas sobre la intención y la con- ducta se han realizado diferentes estudios. En este sentido, Trafimow, Sheeran, Conner y Finlay (2002) realizaron un meta-análisis para cuantificar la relación entre autoeficacia, percepción de control, intenciones y conducta, y encontraron que la autoeficacia fue mejor predictor de la intención y la conducta que la percepción de control.

Resultados similares han sido obtenidos en estudios que se centran en conductas preventivas de salud. Así, Lucidi, Grano, Barbaranelli y Violani (2006) estudiaron la realización de ejercicio en personas mayores y encontraron que la autoeficacia y la percepción de control fueron los mejores predictores de la intención. Por su parte, Tolma, Reininger, Evans y Ureda (2006) obtuvieron que la autoeficacia fue el mejor predictor de la intención de hacerse una mamografía. Myers y Horswill (2006) usaron la TAP para investigar el uso de protección solar en personas que tenían la intención de tomar el sol en verano. Sus resultados mostraron que la autoeficacia fue un predictor significativo tanto de la intención como de la conducta (después de controlar las demás variables de la TAP) mientras que la percepción de control no lo fue. Con una muestra de adolescentes supervivientes de cáncer, Keats, Culos-Red, Courneya y McBride (2006) demostraron que el mejor predictor de la conducta de realización de actividad física fue la autoeficacia, siendo su poder predictivo superior al de la intención. Por último, Heeren, Jemmott, Mandeya y Tyler (2007) en un estudio sobre el uso del preservativo en estudiantes universitarios hallaron que la autoeficacia estuvo relacionada con la intención y el uso del mismo.

En esta misma línea en el presente trabajo se va a investigar utilizando técnicas de modelado causal, si la autoeficacia y la percepción de control presentan diferencias predictivas sobre la intención y las conductas preventivas de la enfermedad cardiovascular. En este sentido, nos planteamos como hipótesis que:

1. La autoeficacia tendrá efectos directos significativos sobre la intención de llevar a cabo conductas preventivas de la enfermedad cardiovascular. 
2. La autoeficacia efectos tendrá directos significativos sobre la ejecución de conductas preventivas de la enfermedad cardiovascular.

3. La percepción de control tendrá efectos directos significativos sobre la intención de llevar a cabo conductas preventivas de la enfermedad cardiovascular y efectos indirectos sobre la ejecución de estas conductas preventivas.

4. La percepción de control tendrá efectos directos significativos sobre las conductas preventivas de la enfermedad cardiovascular.

\section{Metodo}

\section{Descripción de la muestra y procedimiento}

La muestra estuvo integrada por 359 sujetos de ambos sexos de los cuales el $53 \%$ eran mujeres y el $47 \%$ hombres, cuya media de edad era de 41 años, con una desviación típica de 13,42 lo cual indica que la mayor proporción de la muestra recogida se halla en el intervalo de edad en el que empieza a considerarse mayor el riesgo de sufrir algún tipo de enfermedad cardiovascular (Maiques, Brotons \& Villar, 1996; Sanz-Romero, 1996). De ellos el 65, 9\% estaban casados, un 38,2\% tenían estudios universitarios, el 27,4\% estudios medios y también el 27,4\% tenía estudios primarios.

La recogida de la muestra a través de un muestro incidental dirigido, fue realizada por diez entrevistadores entrenados para este fin. Los entrevistadores informaban a los participantes del interés del estudio, solicitando su consentimiento para participar en el mismo. Tras su aceptación respondían al cuestionario, el cual podía ser entregado de forma inmediata o con una dilación temporal de dos días. Se establecieron dos criterios de inclusión para los participantes en el estudio: a) que su edad se situase en torno a los cuarenta años, dado que alrededor de esta edad la manifestación de enfermedad cardiovascular presenta un aumento de frecuencia (Sanz-Romero, 1996); b) que en el momento de la recogida de los datos no se les hubiera detectado ningún trastorno cardiovascular, ya que el objetivo del estudio era conocer el grado de ejecución de ciertas conductas para prevenir la enfermedad cardiovascular. Estas condiciones se preguntaron a los participantes previamente a la cumplimentación del cuestionario y se asumió la sinceridad en las respuestas, dada la garantía de anonimato y el uso de las respuestas para fines de investigación. Los datos obtenidos a este respecto nos indican que el 98\% de la muestra no había sufrido angina de pecho y que el 99\% no había tenido un infarto, ni ninguna enfermedad coronaria. Asimismo, el 97\% de la muestra señaló que no estaban en tratamiento médico habitualmente.

\section{Variables e instrumentos de medida}

El instrumento empleado en este estudio (véase Anexo) fue elaborado por los autores del presente trabajo a partir de los diferentes instrumentos empleados en la literatura revisada y siguiendo las indicaciones realizadas en la Teoría de Acción Planeada (Ajzen, 1985).

Las conductas estudiadas en este trabajo fueron: reducción del consumo de grasas, reducción del consumo de tabaco, realización de ejercicio físico y disminución de los niveles de estrés. El conjunto de estas conductas específicas integran la conducta general de prevención de la enfermedad cardiovascular (Armitage, 2005; Burnout \& Escobar, 2000; Fernández-Abascal et al., 2003; Jhonston et al., 2004). Las respuestas a las escalas están basadas en la percepción que los sujetos poseen acerca de sus creencias y evaluaciones de las mismas. No se han realizado medidas clínicas directas de las variables debido a que los sujetos no participaban en ningún programa de tratamiento, ya que a priori no presentaban ningún síntoma de enfermedad cardiovascular.

$\mathrm{El}$ instrumento empleado estuvo integrado por 16 ítems, con una escala de respuesta tipo Likert de 5 puntos, en la que 1 significaba "estar muy en desacuerdo" y 5 significaba "muy de acuerdo". Para la escala que medía la conducta las alternativas de respuesta iban de "muy infrecuentemente" a "muy frecuentemente".

Cuatro ítems recogían información acerca de la autoeficacia, es decir, la percepción de capacidad para realizar cada una de las conductas que con- 
tribuyen a prevenir la enfermedad cardiovascular. Cada uno de los ítems que medían la autoeficacia fueron definidos como "Me siento capaz de... para prevenir la enfermedad cardiovascular" (Alpha $=.74)$.

Otros cuatro ítems recogían información acerca de la percepción de control, entendida como la facilidad/dificultad para realizar las conductas preventivas citadas teniendo en cuenta las barreras externas. Los ítems fueron definidos del siguiente modo: "Me resulta fácil... para prevenir la enfermedad cardiovascular" (Alpha $=.75$ )

Para medir la intención de conducta se utilizaron cuatro ítems, los cuales fueron formulados del siguiente modo: "Para evitar el riesgo de sufrir una enfermedad cardiovascular tengo la intención de reducir el consumo de ..., o niveles de ... y de realizar ..." (Alpha $=.84)$.

Con objeto de evaluar la conducta actual, se preguntó a los participantes sobre la frecuencia de ejecución de las cuatro conductas de salud estudiadas (Armitage \& Conner, 1999; Godin \& Kok, 1996; Sanz-Romero, 1996). Los ítems fueron formulados en términos de frecuencia de ejecución de la conducta. Ejemplo de ítem: "Para evitar el riesgo de contraer una enfermedad cardiovascular con que frecuencia realiza...". Las alternativas de respuesta iban de muy frecuentemente a muy infrecuentemente (Alpha $=.81)$.

Como puede observarse, los valores Alpha de Crombach de todas las escalas, fueron superiores a .70 , lo cual indica que la consistencia interna de las dimensiones del instrumento es buena (Nunnaly, 1978). Para obtener evidencia de la validez de las escalas, se analizó la estructura factorial del cuestionario mediante un análisis factorial exploratorio. Esta técnica estadística permite resumir la información contenida en un conjunto de variables (ítems) en un número reducido de factores. Los factores representan a las variables originales, con una pérdida mínima de información. Se empleó el método de Componentes Principales con rotación Oblimin con Kaiser para la extracción de los factores, y se retuvieron aquellos factores con eigenvalue mayor que 1.00. (Kaiser, 1960; Tabachnick \& Fidell, 1989). Para asignar los ítems a los factores se consideraron las cargas factoriales iguales o mayores que 0.40 . Se obtuvieron cuatro factores con eigenvalue mayor que 1 , que explicaron $66,93 \%$ de la varianza total. En el Factor I (45,52\% de la varianza) se agruparon los ítems relativos a la intención. En el Factor II (15,65\% de la varianza) se agruparon los ítems relativos a la percepción de control. El Factor III (8,1 \% de la varianza) quedó definido únicamente por dos de los cuatro ítems de la escala de autoeficacia. Por último, los ítems que miden frecuencia de conducta se agruparon en el Factor IV (7,61 \% de la varianza). Por tanto, aunque el factor III necesitaría un mayor evidencia empírica, para este estudio se mantiene la estructura hipotetizada siguiendo la Teoría de Acción Planeada (Ajzen, 1985) ya que en su conjunto los factores explican más del $60 \%$ de la varianza.

\section{Resultados}

Los datos obtenidos se analizaron a través del paquete estadístico de ecuaciones estructurales EQS 6.1 (Bentler, 2006) cuyos resultados comentamos a continuación.

Los resultados de los análisis descriptivos (media y desviación típica) se presentan en la Tabla 1.

\section{TABLA 1}

Media y Desviaciones Típicas de autoeficacia, percepción de control, intención y conducta

\begin{tabular}{lcccc}
\hline & $\begin{array}{c}\text { Autoefi- } \\
\text { cacia }\end{array}$ & $\begin{array}{c}\text { Percepción } \\
\text { de control }\end{array}$ & $\begin{array}{c}\text { Inten- } \\
\text { ción }\end{array}$ & $\begin{array}{c}\text { Con- } \\
\text { ducta }\end{array}$ \\
\hline Media & 3.84 & 3.04 & 3.87 & 3.18 \\
$\begin{array}{l}\text { Desviación } \\
\text { Típica }\end{array}$ & 0.69 & 0.88 & 0.75 & 0.89 \\
\hline
\end{tabular}

Fuente: elaboración propia.

Los índices de ajuste globales del modelo obtuvieron un resultado óptimo (GFI: 0,99; AGFI: 0,97; CFI: 0,99; NFI: 0,99) (véase la Tabla 2).

Además, el Chi cuadrado: 1,28 gl. $p<0,05$ indica un buen ajuste. Por lo que se puede concluir que ajuste general del modelo es satisfactorio. Por otra parte, se calcularon los índices de parsimonia del modelo (PGFI: 0.165; PNFI: 0.166). En 
TABLA 2

Índices globales del modelo

\begin{tabular}{lcc}
\hline \multicolumn{1}{c}{ Índices } & $\begin{array}{c}\text { Valor } \\
\text { Óptimo }\end{array}$ & $\begin{array}{c}\text { Valor } \\
\text { Obtenido }\end{array}$ \\
\hline $\begin{array}{l}\text { Índice de bondad de ajuste GFI } \\
\text { (Goodness of Fit Index) }\end{array}$ & $>.90$ & .99 \\
$\begin{array}{l}\text { Índice de ajuste ajustado AGFI } \\
\text { (Adjusted Goodness of Fit Index) }\end{array}$ & $>.90$ & .97 \\
$\begin{array}{l}\text { Índice de ajuste comparativo } \\
\text { CFI (Comparative Fit Index) }\end{array}$ & $>.90$ & .99 \\
$\begin{array}{l}\text { Índice de ajuste normalizado } \\
\text { NFI (Normed Fit Index) }\end{array}$ & $>.90$ & .99 \\
\hline
\end{tabular}

Fuente: elaboración propia.

ambos índices valores superiores a 0.50 indican un ajuste parsimonioso óptimo. Por lo que, según estos resultados, el modelo no sería parsimonioso. Para que el modelo fuera más parsimonioso se deberían eliminar relaciones. Sin embargo, dado que las relaciones incluidas en el modelo tienen un efecto significativo, se ajustan a la teoría expuesta y el modelo posee un ajuste global satisfactorio, no se considera pertinente llevar a cabo dicha eliminación.

Se confirman estadísticamente tres de las cuatro hipótesis planteadas. Así, tal como se muestra en la Figura 1, se puede observar que la autoeficacia influye de manera positiva y significativa sobre la intención con un Coeficiente Estructural
Estandarizado (CEE) de 0,57 $(p<0,01)$ y sobre la Conducta (CEE de 0,32; $p<0,01$ ). Por su parte, la percepción de control presenta una influencia negativa y significativa sobre la intención (CEE de $-0,16 ; p<0,01)$. Finalmente, la intención muestra un efecto positivo y significativo sobre la conducta (CEE de 0,37; $p<0,01$ ). Sin embargo, la percepción de control no tiene un efecto directo significativo sobre la conducta.

Además se observa una correlación positiva y significativa $(0,14 ; p<0,01)$ entre las dos variables externas (autoeficacia y percepción de control).

Con estos resultados el modelo permite predecir el 33\% de la varianza explicada de la intención y el 38\% de la varianza explicada de la conducta.

\section{Discusión y conclusiones}

El objetivo de este trabajo ha sido analizar si la autoeficacia y la percepción de control presentan diferencias predictivas sobre la intención y las conductas preventivas de la enfermedad cardiovascular. Los análisis realizados han permitido constatar dichas diferencias, siendo la autoeficacia el mejor predictor de la intención y de la conducta, en congruencia con los resultados obtenidos en diferentes investigaciones relacionadas con otras conductas de salud (Heeren et al., 2007; Keats et al., 2006; Lucidi et al., 2006; Myers \& Horswill, 2006; Terry, 1993; Terry \& O'Leary, 1995; Tolma et al., 2006; Trafimow et al., 2002). Es decir, cuando la persona

\section{FIGURA 1}

Efectos de la autoeficacia y percepción de control sobre la intención y la conducta

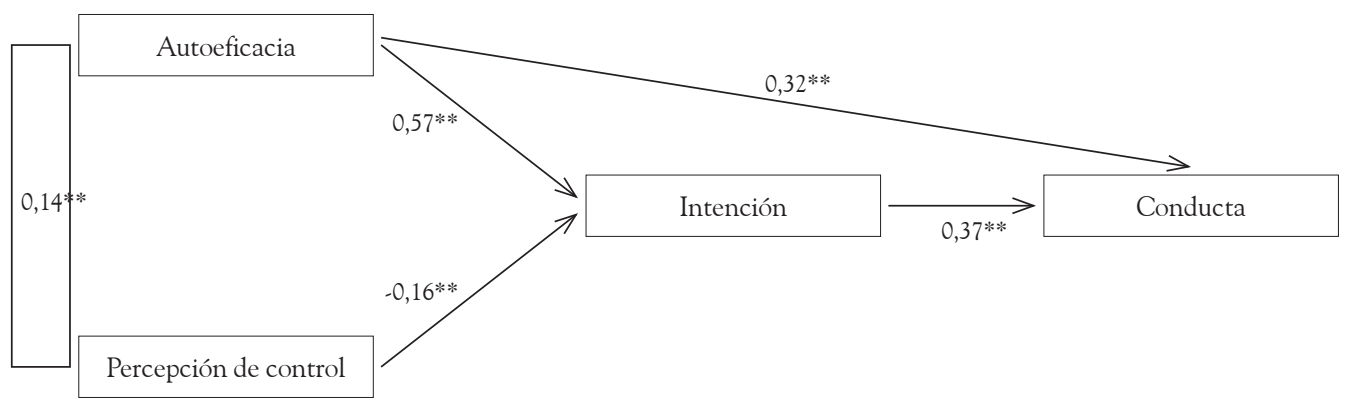

$* * p>0,01$.

Fuente: elaboración propia. 
se siente capaz de realizar una acción tendrá una mayor intención de realizarla en un futuro próximo y será más probable que la lleve a cabo.

Sin embargo, a pesar de que una alta percepción de autoeficacia pueda llevar a una mayor intención y ejecución de la conducta, en muchos casos la presencia de barreras o limitaciones externas a la persona (las decisiones de otras personas, la falta de oportunidades, etc.) pueden influir también sobre la intención de realizar dicha conducta. En este sentido, la percepción de control, medida como la facilidad/dificultad percibida para llevar a cabo un determinado comportamiento, obtiene en nuestro estudio una influencia directa y negativa aunque más débil en el desarrollo de la intención. Es decir, cuantos menos obstáculos se presenten en una situación más fácil será que se desarrolle la intención de conducta, aunque no la conducta misma, en congruencia con los resultados obtenidos por Ryn, Lytle y Kirscht (1996) y Michels y Kugler (1998).

Los resultados del presente trabajo son importantes ya que muestran, como los obtenidos por Trafimow et al. (2002), la conveniencia de distinguir entre autoeficacia (a la cual denominan dificultad percibida) y percepción de control, dentro de la TAP. El hecho de que ambos constructos tengan diferentes niveles de relación con la intención y la conducta, apoya la validez de tal distinción que ha sido encontrada en trabajos previos y que se extiende, con este trabajo, a la prevención de la enfermedad cardiovascular.

No obstante todavía sería necesario dar un paso más en el estudio de conductas de salud. En esta línea Conner (2008) señala que la mayoría de los modelos de salud (por ejemplo, los modelos de fases) se centran en el inicio de las conductas pero no en su mantenimiento. Así, para algunas conductas de salud como las aquí estudiadas la iniciación es necesaria pero no suficiente, para que se produzcan beneficios sobre la salud. Para tales conductas de mantenimiento, serán necesarios estudios en los que se analicen las variables que influyen tanto en el inicio como en el mantenimiento de conductas saludables prolongadas en el tiempo. En este sentido, la autoeficacia ha sido señalada como una variable importante para el inicio y mantenimiento de la conducta (Conner, 2008), aunque se debería profundizar en el estudio de sus diferentes formas de actuación.

Además, es necesario subrayar que la realización de conductas preventivas y de promoción de salud dependen no sólo de características individuales de la persona, sino también de factores culturales y sociopolíticos, siendo necesario tenerlos en cuenta para comprender las dificultades encontradas en la ejecución de los cambios de hábitos, que permitan promover la salud en general y en particular la cardiovascular (Parales, 2006).

Por otra parte, es de destacar el buen ajuste del modelo analizado a nivel global en nuestra muestra de estudio. No obstante, es necesario reconocer que el diseño transversal de este estudio limita los resultados obtenidos, ya que la mejor forma de establecer la relación entre las variables estudiadas es con diseños longitudinales. Además, dado que la muestra de estudio se ha limitado a un sector concreto de la población, los resultados no se pueden considerar generalizables y debería replicarse con distintas muestras.

Desde este trabajo, no sólo se confirma la necesidad de diferenciar a nivel conceptual y operacional entre la percepción de control y las creencias de autoeficacia como determinantes relevantes de la intención de realizar las conductas preventivas de salud, sino también su utilidad práctica como guía para el diseño de programas de educación y promoción de la salud. De hecho, una de las prioridades de la OMS ha sido modificar hábitos alimenticios y sedentarios en la población, ya que promocionar la adopción de hábitos saludables puede incidir de forma significativa en la reducción de las consecuencias vitales, económicas y afectivas que conllevan las distintas cardiopatías.

En el marco de la prevención primaria, es especialmente relevante conocer si las dificultades para la acción se encuentran en los obstáculos y/o barreras externas, o bien se deben a la necesidad de desarrollar las habilidades oportunas y la confianza en las mismas, para que realmente puedan realizarse los cambios comportamentales que potencien la salud. En concreto, en la prevención de la enfermedad cardiovascular, nuestros resultados 
sugieren que las acciones deberían dirigirse sobre todo al desarrollo de un fuerte sentido de autoeficacia (percepción de capacidad) en la persona debido a su influencia directa sobre la intención y la ejecución de las conductas preventivas.

Por otra parte, como sugieren diversos autores (Ajzen, 1991; Pastor, Pons; López-Roig \& MartínAragón, 2000; Villamarín \& Álvarez, 1998), dada la complejidad del comportamiento humano sería interesante incluir otras variables en la predicción de comportamientos de salud como la etapa o fase en la que se encuentra la persona en su proceso de cambio (Prochaska, 1993) o el comportamiento previo y/o hábito (Carpi et al., 2007; Norman \& Conner, 1995) y, sobre todo, la unificación de criterios respecto a la definición y operacionalización de las variables que integran los modelos teóricos que intentan predecir las conductas relacionadas con la salud.

En suma, el presente trabajo ha mostrado la conveniencia de distinguir entre autoeficacia y percepción de control en la TAP en la prevención de conductas de salud cardiovascular, y también ha destacado la importancia predictiva de la autoeficacia sobre la intención y las conductas estudiadas.

\section{Referencias}

Ajzen, I. \& Fisbein, M. (1980). Understanding attitudes and predicting social behaviour. Englewood-Cliffs, NJ: Prentice-Hall.

Ajzen, I. (1985). From intentions to actions: A Theory of Planned Behavior. En J. Khul, \& J. Beckmann (Eds), Action control from cognition to behavior (pp. 10-39). Germany: Springer-Verlag. 31.

Ajzen, I. (1988). Attitudes, Personality and Behavior. Chicago: The Dorsey Press.

Ajzen, I. (1991). The theory of planned behavior. Organitational Behavior and Human Decision Proceses, 50, 179-211.

Ajzen, I. \& Madden, T. J. (1985). Prediction of goal-directed behavior: Attitudes, intentions and perceived behavioral control. Journal of Experimental Social Psychology, 22, 453-474.

Albarracín, D., Johnson, B. T., Fishbein, M. \& Muellerleile, P. A. (2001). Theories of reasoned action and planned behaviour as models of condom use: A meta-analysis. Psychological Bulletin, 127, 142-161.

Armitage, C. (2005). Can the theory of planned behavior predict the maintenance of pshysical activity? Health Psychology, 24, 235-245.

Armitage, C. J. \& Conner, M. (1999). Predictive validity of the Theory of Planed Behaviour: The role of questionnaire format and social desirability. Journal of Community and Applied Social Psychology, 9, 261-272.

Armitage, C. J. \& Conner, M. (1999). Efficacy of the theory of planned behaviour: A meta-analytic review. British Journal of Social Psychology, 40, 471-499.

Bandura, A. (1977). Self-Efficacy: Toward a unifying theory of behavioral change. Psychological Review, 84, 191-215.

Bentler, P. M. (2006). EQS 6.1 for Windows. Encino, CA: Multivariate Software Inc.

Brenes, G., Strube, M. \& Storandt, M. (1998). An application of the Theory of Planned Behaviour to exercise older adults. Journal of Applied Social Psychology, 28, 2274-2290.

Burnout, D. \& Escobar, E. (2000). Prevención de enfermedades cardiovasculares: iDeben aplicarse los mismos criterios en América Latina que en Europa y Norteamérica? Revista Española de Cardiología, 53, 889-895.

Carpi, A., Zurriaga, R., González, P., Marzo, J. C. \& Buunk, A. P. (2007). Incidencia de los hábitos de conducta en la prevención de la enfermedad cardiovascular. International Journal of Clinical and Health Psychology, 7, 57-90.

Conner, M. (2008). Initiation and maintenance of health behaviours. Applied Psychology: An International Review, 57, 42-50.

Conner, M., Sandberg, T., McMillan, B. \& Higgins, A. (2006). Role of anticipated regret in adolescent smoking initiation. British Journal of Health Psychology, 11, 85-101.

Corella, D. \& Ordovás, J. M. (2007). Genes, dieta y enfermedades cardiovasculares. Investigación y Ciencia, Noviembre, 74-83.

Courneya, K. S., Conner, M. \& Rhodes, R. E. (2006). Effects of different measurement scales on the variability and predictive validity of the "two-com- 
ponent" model of the theory of planned behaviour in the exercise domain. Psychology and Health, 21(5), 557-570.

DeVellis, B. M., Blalock S. J. \& Sandler, R. (1990). Predicting participation in cancer screening: The role of perceived behavioral control. Journal of Applied Social Psychology, 20, 639-660.

Fernández-Abascal, E., Martín, M. D. \& Domínguez, F. J. (2003). Factores de riesgo e intervenciones psicológicas eficaces en los trastornos cardiovasculares. Psicothema, 15, 615-630.

Fishbein, M. \& Ajzen, I. (1975). Belief, attitude, intention and behavior: An introduction to Theory and Research. Massachusetts: Addison-Wuesly Publishing Company.

Godin, G. \& Kok, G. (1996). The theory of planned behavior: A review of its applications to healthrelated behaviors. American Journal of Health Promotion, 1, 87-98.

Guerrero, C. \& Palmero, F. (2006). Percepción de control y respuestas cardiovasculares. International Journal of Clinical and Health Psychology, 6, 145-168.

Heeren, G. A., Jemmott, J. B., Mandeya, A. \& Tyler, J. C. (2007). Theory-based predictors of condom use among university students in the United States and South Africa. AIDS Education and Prevention, 19(1), 1-12.

Holguín, L., Correa, D., Arrivillaga, M., Cáceres, D. \& Varela, M. (2006). Adherencia al tratamiento de hipertensión arterial: efectividad de un programa de intervención biopsicosocial. Universitas Psychologica, 5(3), 535-547.

Johnston, D. W., Johnston, M., Pollard, B., Kinmonth, A. L. \& Mant, D. (2004). Motivation in not enough: Prediction of risk behavior following diagnosis of coronary heart disease from the Theory of planned behavior. Health Psychology, 23, 533-538.

Kaiser, H. F. (1960). The application of electronic computers to factor analysis. Educational and Psychological Measurement, 20141-151.

Keats, M. R., Culos-Red, S. N., Courneya K. S. \& McBride, M. (2006). Understanding physical activity in adolescent cancer survivors: An application of the theory of planned behavior. Psycho-Oncology, 16, 448-457.
Lucidi, F., Grano, C., Barbaranelli, C. \& Violani, C. (2006). Social-cognitive determinants of physical activity attendance in older adults. Journal of Aging and Physical Activity, 14(3), 344-359.

Maiques, A., Brotons, C. \& Villar, F. (1996). Guía de prevención cardiovascular. Madrid: Grupo MSD. Merck Sharp \& Dohme de España, S.A.

Michels, T. C. \& Kugler, J. P. (1998). Predicting exercise in older Americans: Using the theory of planned behavior. Military Medicine, 163, 524-529.

Montero, I. \& León, O. G. (2005). Sistema de clasificación del método en los informes de investigación en Psicología. International Journal of Clinical and Health Psychology, 5, 115-127.

Myers, L. B. \& Frost, S. (2002). Smoking and smoking cessation: Modifying perceptions of risk? En D. Futter \& L. Quine (Eds), Changing Health Behaviour: Research and practice with Social Cognition Models (pp. 49-65). Milton Keynes, UK: Open Universtiy Press.

Myers, L. B. \& Horswill, M. S. (2006). Social cognitive predictors of sun protection intention and behavior. Behavioral Medicine, 32, 57-63.

Nunnaly, N. C. (1978). Psychometric theory. Nueva York: McGraw-Hill.

Panday, S., Reddy, S. P., Ruiter, R. A., Bergström, E. \& De Vries, H. (2007). Determinants of smoking among adolescents in the Southern Cape-Karoo region, South Africa. Health Promotion International, 22(3), 207-217.

Parales Quenza, C. J. (2006). Representaciones sociales de comer saludablemente: un estudio empírico en Colombia. Universitas Psychologica, 5 (3), 613-626.

Pastor, M. A., Pons, N., López-Roig, S. \& MartínAragón, M. (2000). Aproximación psicosocial al estudio de la discapacidad: aportaciones desde la Teoría de la Acción Planeada. Revista de Psicología Social Aplicada, 10, 81-96.

Prochaska, J. (1993). Modelo transteórico de cambio para conductas adictivas. En M. Caas \& M. Gossop (Eds), Recaída y prevención de recaídas. Tratamientos psicológicos en drogodependencias (pp. 85-136). Barcelona: Ediciones en Neurociencias.

Sanz-Romero, G. A. (1996). Cardiopatía isquémica. En P. Valentí (Ed.), Medicina interna (pp. 533-552). Barcelona: DOYMA. 
Tabachnick, B. G. \& Fidell, L. S. (1989). Using multivariate statistics. Nueva York: Harper Collins.

Terry, D. J. (1993). Self-efficacy expectancies and the theory of reasoned action. En D. J. Terry \& C. Gallois (Eds), The theory of reasoned action: Its application to AIDS-preventive behaviour. International series in experimental social psychology (Vol. 28, pp. 135-151). Oxford, England UK: Pergamon Press.

Terry, D. J. \& O'Leary, J. E. (1995). The theory of planned behaviour: The effects of perceived behavioural control and self-efficacy. British Journal of Social Psychology, 34, 199-220.

Tolma, E., Reininger, B., Evans, A. \& Ureda, J. (2006). Examining the Theory of Planned Behavior and the construct of Self-Efficacy to predict mammography intention. Health Education \& Behavior, 33(2), 233-251.

Trafimow, D., Sheeran, P., Conner, M. \& Finlay, K. A. (2002). Evidence that perceived behavioral control is a multidimensional construct: Perceived control o perceived difficulty. British Journal of Social Psychology, 41, 101-121.

Villamarín F. \& Álvarez, M. (1998). Modelos sociocognitivos en promoción de la salud: un análisis conceptual. Psicologemas, 12, 161-203.

World Health Organization. (2009). Disponible en http://www.who.int/healthinfo/global_burden_ disease/GlobalHealthRisks_report_full.pdf

\section{Anexo}

\section{Ítems del cuestionario utilizado}

1. Me siento capaz de disminuir el consumo de tabaco para prevenir una enfermedad cardiovascular.

2. Me siento capaz de disminuir el consumo de grasas animales para prevenir una enfermedad cardiovascular.
3. Me siento capaz de disminuir los niveles de estrés para prevenir una enfermedad cardiovascular.

4. Me siento capaz de realizar ejercicio físico para prevenir una enfermedad cardiovascular.

5. Me resulta fácil disminuir el consumo de tabaco para prevenir una enfermedad cardiovascular.

6. Me resulta fácil disminuir el consumo de grasas animales para prevenir una enfermedad cardiovascular.

7. Me resulta fácil disminuir los niveles e estrés para prevenir una enfermedad cardiovascular.

8. Me resulta fácil realizar ejercicio físico para prevenir una enfermedad cardiovascular.

9. Para evitar el riesgo de sufrir una enfermedad cardiovascular tengo la intención de reducir el consumo de tabaco.

10.Para evitar el riesgo de sufrir una enfermedad cardiovascular tengo la intención de reducir el consumo de grasas animales.

11.Para evitar el riesgo de sufrir una enfermedad cardiovascular tengo la intención de realizar ejercicio físico.

12.Para evitar el riesgo de sufrir una enfermedad cardiovascular tengo la intención de disminuir los niveles de estrés.

13. Para evitar el riesgo de contraer una enfermedad cardiovascular, icon qué frecuencia, en la actualidad, disminuye el consumo de tabaco?

14. Para evitar el riesgo de contraer una enfermedad cardiovascular, icon qué frecuencia, en la actualidad, disminuye el consumo de grasas animales?

15.Para evitar el riesgo de contraer una enfermedad cardiovascular, icon qué frecuencia, en la actualidad, disminuye los niveles de estrés?

16.Para evitar el riesgo de contraer una enfermedad cardiovascular, icon qué frecuencia, en la actualidad, realiza ejercicio físico? 